[Original]

\title{
Changes in Presenteeism Six Months After Returning from Sick Leave Due to Mental Illness
}

\author{
Akira Kusumoto $^{1 *}$, Shigeyuki KaJiki ${ }^{1}$, Tomomi Anan ${ }^{1}$, Tomohisa Nagata $^{1}$, Masako Nagata ${ }^{1}$, \\ Yoshihisa FuJINO ${ }^{2}$ and Koji Mori ${ }^{1}$ \\ ${ }^{1}$ Department of Occupational Health Practice and Management, Institute of Industrial Ecological Sciences, \\ University of Occupational and Environmental Health, Japan. Yahatanishi-ku, Kitakyushu 807-8555, Japn \\ ${ }^{2}$ Department of Environmental Epidemiology, Institute of Industrial Ecological Sciences, \\ University of Occupational and Environmental Health, Japan. Yahatanishi-ku, Kitakyushu 807-8555, Japn
}

\begin{abstract}
This study examines how psychological distress (measured by the K10 screening test) and presenteeism (measured by the quality and quantity method) change in the six months after returning to work from having taken a sick leave because of a mental illness. In a manufacturing company with approximately 2,600 employees, 23 employees returned to work after experiencing mental illness between April 2015 and March 2016, and all 23 agreed to participate in the study. We analyzed 18 cases for which we had sufficient data. Two of the employees were absent from work in the sixth month. We performed multilevel analysis for K10 and presenteeism over time on the 16 without recurrence. A significant decreasing trend was observed for both K10 and presenteeism. Eleven of the 16 employees were consistently below the K10 cutoff value of 10 for six months, and 5 had zero presenteeism in the sixth month, whereas 6 employees showed improvement in presenteeism that stopped midway through the study. An occupational physician judged that the employees could work normally with presenteeism of zero. After returning to work, it is important to monitor not only psychiatric symptoms but also presenteeism.
\end{abstract}

Keywords : mental health, presenteeism, return to work, work accommodation.

(Received January 19, 2021, accepted July 20, 2021)

\section{Introduction}

An increase in presenteeism has attracted attention in recent years, as mental illness-related issues in the workplace, and the associated economic loss, has been reported as substantial $[1,2]$. Wada et al found that labor losses related to mental illness were high in Japan [3], and Nagata et al reported substantial mental illness-related productivity losses [4].

With increased rates of mental illness, the number of employees taking sick leave has also risen. The Jap- anese government has developed guidelines for workplaces and occupational physicians (OPs) regarding the assessment of return to work (RTW) following sick leave for mental illness [5]. These guidelines specify that psychiatrists should evaluate whether a worker's symptoms have sufficiently improved to allow RTW, and OPs should evaluate whether it is appropriate from the perspective of the workplace. Consequently, the employer makes the final decision, based on the OP's opinion, about the employee's RTW. After RTW, in addition to observation and support by the supervisor,

*Corresponding Author: Akira Kusumoto, Department of Occupational Health Practice and Management, Institute of Occupational Ecological Sciences, University of Occupational and Environmental Health, Japan. 1-1 Iseigaoka, Yahatanishi-ku, Kitakyushu 807-8555, Japan, Tel: +81-93-6917523, Fax: +81-93-692-4590, E-mail: yokaniseakira@med.uoeh-u.ac.jp 
the guidelines also suggest that a follow-up be conducted by occupational health staff in the workplace, and that a plan for RTW be evaluated and reviewed as appropriate.

OPs generally confirm the conditions of both mental health and fitness for work after RTW. From the perspective of preventing relapse after RTW, it has recently been pointed out that it is important to focus not only on psychiatric symptoms but also on social adaptation and cognitive functions [6], but it is not practical to follow up social adaptation and cognitive functions every month due to the complexity and time constraints.

There is a way for OPs to use presenteeism as a measure to follow up the condition of workers after RTW regularly, apart from psychiatric symptoms. Presenteeism is defined as "a state of decreased productivity due to poor physical or health condition". Various methods have been developed in previous research to measure presenteeism as decreased productivity $[7,8]$. As all those methods are self-administered questionnaires, Johns suggested that a theory of presenteeism should recognize the subjectivity of health [9]. $\mathrm{He}$ pointed out that any theory in this domain must recognize the essential subjectivity of people's evaluation of their own health status and accommodate well-established individual differences in the propensity for self-disclosure of chronic illness at work, perceptions of how work affects health, and the tendency to adopt a sick role. Various studies of presenteeism are being conducted, despite the limitations of subjective evaluation $[9,10]$.

Fujino et al reported that previous presenteeism questionnaires appeared to have captured productivity but to have unintentionally ignored the distinction between productivity and the ability to function at work [11]. The terms "functioning" and "performance" are used to mean productivity in the papers cited here, and are cited as is. Mental illness commonly causes presenteeism, which can persist in terms of decreased productivity, even if symptoms improve. Adler et al reported that the job performance of employees who had been on mental health leave was consistently lower than that of controls at 18 months follow-up, even if depression symptoms had improved [12]. Curkendall et al showed that, even when depressed patients were treated with anti-depressants, there were substantial productivity losses [13]. Gilmour et al noted that impaired work functioning may persist even after achieving remission from depression [14].

Presenteeism may therefore be a useful indicator for OPs to assess the degree of fitness for work after RTW, but, to the best of our knowledge, no previous studies have investigated how psychiatric symptoms and presenteeism change after RTW following mental health leave. Despite OPs confirming the employees' psychiatric symptoms and providing their opinion on the appropriate workload after RTW, the relationship between the OP's opinion and presenteeism remains unclear. To clarify this relationship, the present study sought to investigate the following:

1. Changes in monthly psychological distress.

2. Changes in monthly presenteeism in workers with mental illness during the first six months after RTW.

3. The relationship between the OP's opinions and presenteeism.

\section{Subjects and Methods}

\section{Study design}

This was a prospective study. We used a self-administered questionnaire survey.

\section{Study setting}

The study was conducted at a manufacturing company that employed approximately 2,600, where workers could take up to one year of sick leave. The occupational health team consisted of one dedicated $\mathrm{OP}$ and three dedicated public health nurses. Generally, the dedicated OP interviewed employees with mental illness every month for six months after RTW, and continued to interview them for longer periods if necessary. The dedicated OP had three years of experience as an OP at the start of this study. The study period was between April 2015 and March 2016.

\section{Monitoring after returning to work}

After a worker submitted a medical certificate of permission to return to the workplace, the dedicated OP interviewed the worker to determine whether they were able to RTW. During the interview, the OP explained the present study to the worker, that it was 
investigating how psychological distress and presenteeism change during six months after RTW. The dedicated OP explained to the employees that they were free to choose to participate in the study, then obtained their consent to participate. If the worker consented to participate in the study, they were asked to fill out a questionnaire survey, which collected demographic information such as their sex, age (20s, 30s, 40s), occupational type (manufacturing/office), employment status, position, educational background (graduation from high school/junior college, college of technology/university, graduate school), marital status, smoking status, drinking status, physical illness, years of service, days of sick leave before RTW, and the number of times of sick leave taken. The OP collected selfadministered questionnaires on psychological distress and presenteeism in monthly interviews, when the OP evaluated whether the work burden was at a level that would exacerbate the psychiatric symptoms.

Norder et al suggested that occupational and primary health care providers should consider reviewing the mental health status of workers six months after recovery from a mental health-related absence. Following this recommendation, we used a study period of six months [15].

\section{Subjects}

Of the workers who had been on leave due to mental illness during the study period, 23 had submitted a medical certificate to the workplace permitting RTW. All 23 agreed to participate in the study. One subject received extended leave without RTW. Four subjects were excluded because data had not been collected for more than two months. Finally, we surveyed 18 workers who returned to work after having been on leave for mental illness.

\section{Psychological distress}

The survey was designed to include various kinds of mental illness, such as depression and anxiety, as well as cases that had been cured. We decided to monitor psychological distress, not psychiatric symptoms directly, to monitor the mental state of the patients. The $\mathrm{K} 10$ is a self-report questionnaire that quantifies nonspecific psychological distress in general and screens for any DSM-IV depressive and anxiety disorders, with a total of 10 questions, each rated from 0 to 4 points, giving a maximum total score of 40 points [16]. The Japanese version of the K10 has been tested [17], and a cutoff value of 10 or more is considered to indicate a mood disorder/anxiety disorder. Each participant completed the K10 during an interview with the OP every month for six months after RTW.

\section{Presenteeism}

The quality and quantity (QQ) method was used to measure presenteeism. We asked employees about the quality/quantity of work that they were able to perform when suffering from their symptoms, compared to when they were not. Responses were given on a scale from 0 (no productivity) to 10 (no productivity loss) $[4,18]$.

Presenteeism $=1-$ Quantity $(0-10) *$ Quality $(0-10) / 100$

\section{Diagnosis}

The name of the disease was adopted from the psychiatrist's medical certificate that the worker submitted to the workplace when RTW.

\section{Presence of the OP's written opinion}

We checked whether the OP had provided a written opinion regarding work restrictions in the workplace, such as recommending no overtime or no travel.

\section{Statistical analysis}

$\mathrm{K} 10$ and presenteeism over time were analyzed by multilevel analysis in the participants, excluding those who took another period of sick leave during the study period. In each case, we analyzed whether there was a difference in the overall results from month to month, regarding the months as continuous variables. The first month was used as a reference and compared with each month, regarding the months as categorical variables. A statistical significance was set as $P<0.05$. Data analyses were performed using Stata version 16 software (STATA Corp, Texas USA).

\section{Ethics approval}

The study was explained verbally and in writing to the participating employees and the manufacturing company by a collaborating OP. Employees were free 
to choose to participate or not. Employers were not allowed to view the employees' self-reported questionnaire responses. This survey was approved by the Ethics Committee of the University of Occupational and Environmental Health (H26-088). All obtained data were anonymized.

\section{Results}

\section{Demographic data}

Of the 18 employees, 15 were male and 3 were female, all of whom had regular non-managerial roles: 2 manufacturing employees and 16 clerical employees. The types of mental illness among the 18 employees were as follows: 12 participants had depression, 5 had adaptation disorders, and 1 had an anxiety disorder (Table 1). Of these, two male employees (case 17, 18) took another period of sick leave in the six months after RTW. They submitted medical certificates from their doctors saying that they needed time off from work; thus, we were unable to obtain their data in the sixth month because they went on leave upon submission of the certificates. In both cases, the diagnosis was depression. In terms of the number of sick leaves, it was the first time for case 17 and the third time for case 18 to RTW.

The average length of service was 12.9 years, with an average of 184.9 days off before RTW. Before the investigation, six of the employees had taken sick leave prior to the current period of leave, with an average of 1.5 periods of leave (Table 2).

\section{Monthly changes in psychological distress}

Throughout the six months, the average scores were below the cutoff value of 10 [15] (Table 3). Of the 16 employees who did not take another period of leave, 11 (cases 1, 2, 3, 5, 8, 10, 11, 12, 13, 14, and 15) did not exceed the cutoff in the six months, whereas 5 (cases 4, 6, 7, 9, and 16) exceeded the cutoff at least once in the 6-month period (Figure 1). K10 over time was analyzed by multilevel analysis for the 16 employees. It showed a significant difference in the overall change in monthly decrease (Coefficient $=-0.29, P=0.019$ ) (Table 4), but no significant differences were found between the first month and each month except for the fourth month (Coefficient $=-1.94, P=0.006)$ (Table 4).
Table 1. Participants' characteristics

\begin{tabular}{|c|c|c|}
\hline & $\mathrm{n}$ & $\%$ \\
\hline \multicolumn{3}{|l|}{ Sex } \\
\hline Men & 15 & 83 \\
\hline Women & 3 & 17 \\
\hline \multicolumn{3}{|l|}{ Age (years) } \\
\hline $20-29$ & 3 & 17 \\
\hline $30-39$ & 9 & 50 \\
\hline $40-49$ & 6 & 33 \\
\hline \multicolumn{3}{|l|}{ Diagnosis } \\
\hline Depression & 12 & 67 \\
\hline Adaptation disorder & 5 & 28 \\
\hline Anxiety disorder & 1 & 6 \\
\hline \multicolumn{3}{|l|}{ Occupation type } \\
\hline Manufacturing & 2 & 11 \\
\hline Office & 16 & 89 \\
\hline \multicolumn{3}{|l|}{ Education background } \\
\hline High school & 5 & 28 \\
\hline Junior college, college of technology & 4 & 22 \\
\hline University, graduate school & 8 & 44 \\
\hline Missing & 1 & 6 \\
\hline \multicolumn{3}{|l|}{ Marital status } \\
\hline Single & 12 & 67 \\
\hline Married & 6 & 33 \\
\hline \multicolumn{3}{|l|}{ Smoker } \\
\hline Yes & 8 & 44 \\
\hline No & 10 & 56 \\
\hline \multicolumn{3}{|l|}{ Alcohol drinker } \\
\hline Often & 10 & 56 \\
\hline Occasionally & 7 & 39 \\
\hline Missing & 1 & 6 \\
\hline \multicolumn{3}{|l|}{ Physical illness } \\
\hline Yes & 2 & 11 \\
\hline No & 16 & 89 \\
\hline \multicolumn{3}{|l|}{ Additional period of leave } \\
\hline Yes & 2 & 11 \\
\hline No & 16 & 89 \\
\hline
\end{tabular}


Table 2. Participants' characteristics

\begin{tabular}{lll}
\hline & $\mathrm{n}=18$ & \\
\cline { 2 - 3 } & Mean & Standard Deviation \\
\hline Years of service & 12.9 & 7.7 \\
Days of sick leave before returning to work & 184.9 & 126.5 \\
The number of times of sick leave taken & 1.5 & 0.8 \\
\hline
\end{tabular}

Table 3. Participants' changes in K10 and presenteeism $(n=18)$

\begin{tabular}{|c|c|c|c|c|c|c|c|}
\hline & Mean & $\begin{array}{l}\text { Standard } \\
\text { Deviation }\end{array}$ & Minimum & 25 th percentile & Median & 75th percentile & Maximum \\
\hline \multicolumn{8}{|l|}{ K10 } \\
\hline at 1 month & 6.28 & 6.07 & 0.00 & 1.00 & 4.50 & 11.00 & 19.00 \\
\hline at 2 months & 6.00 & 5.38 & 0.00 & 2.00 & 4.00 & 10.00 & 19.00 \\
\hline at 3 months & 4.94 & 5.32 & 0.00 & 1.00 & 3.00 & 6.00 & 19.00 \\
\hline at 4 months & 3.44 & 5.02 & 0.00 & 0.00 & 2.00 & 4.00 & 17.00 \\
\hline at 5 months & 4.56 & 5.58 & 0.00 & 0.00 & 3.00 & 5.00 & 17.00 \\
\hline at 6 months* & 4.31 & 5.78 & 0.00 & 0.00 & 1.50 & 5.00 & 18.00 \\
\hline \multicolumn{8}{|l|}{ Presenteeism } \\
\hline at 1 month & 0.58 & 0.28 & 0.00 & 0.51 & 0.52 & 0.80 & 0.97 \\
\hline at 2 months & 0.47 & 0.20 & 0.19 & 0.36 & 0.44 & 0.62 & 0.84 \\
\hline at 3 months & 0.36 & 0.19 & 0.10 & 0.19 & 0.32 & 0.44 & 0.76 \\
\hline at 4 months & 0.32 & 0.23 & 0.00 & 0.19 & 0.28 & 0.51 & 0.75 \\
\hline at 5 months & 0.31 & 0.26 & 0.00 & 0.19 & 0.28 & 0.44 & 0.98 \\
\hline at 6 months* & 0.26 & 0.27 & 0.00 & 0.00 & 0.19 & 0.37 & 0.96 \\
\hline
\end{tabular}

Presenteeism $=1-$ Quantity $(0-10) *$ Quality $(0-10) / 100$

*: Calculated with 16 people, excluding two leavers

Table 4. Changes in $\mathrm{K} 6$ after returning from sick leave due to mental illness

\begin{tabular}{lllllll}
\hline & & Coefficient & Standard Error & $95 \%$ Confidence Interval & $P$ \\
\hline month(continuous) & & -0.29 & 0.12 & -0.53 & -0.05 & 0.019 \\
month(categorical) & 1 & reference & & & & \\
& 2 & 0.25 & 0.36 & -1.13 & 1.63 & 0.722 \\
& 3 & -0.81 & -1.16 & -2.19 & 0.57 & 0.248 \\
& 4 & -1.94 & -2.76 & -3.32 & -0.56 & 0.006 \\
& 5 & -1.31 & -1.87 & -2.69 & 0.07 & 0.062 \\
& 6 & -0.88 & -1.24 & -2.25 & 0.50 & 0.213 \\
\hline
\end{tabular}

\section{Monthly changes in presenteeism}

The average presenteeism in the 18 participants in the first month after RTW was 0.58 , and presenteeism at the sixth month (based on the results of 16 participants, excluding the 2 who were on sick leave again) was 0.26 (Table 3 ). Presenteeism over time was analyzed by multilevel analysis in 16 employees. It showed a significant difference in the overall change in monthly decrease (Coefficient $=-0.06, P=0.000$ ) (Table 5). Presenteeism in all the months except for the second month was significantly lower than in the first month (Coefficient $=-0.19, P=0.000$ at the $3 \mathrm{rd}$ month, Coefficient $=-0.26, P=0.000$ at the 4 th month, Coefficient $=-0.26, P=0.000$ at the 5 th month, Coefficient $=-0.29, P=0.000$ at the 6 th month) (Table 5).

Of the 11 employees who never exceeded the K10 


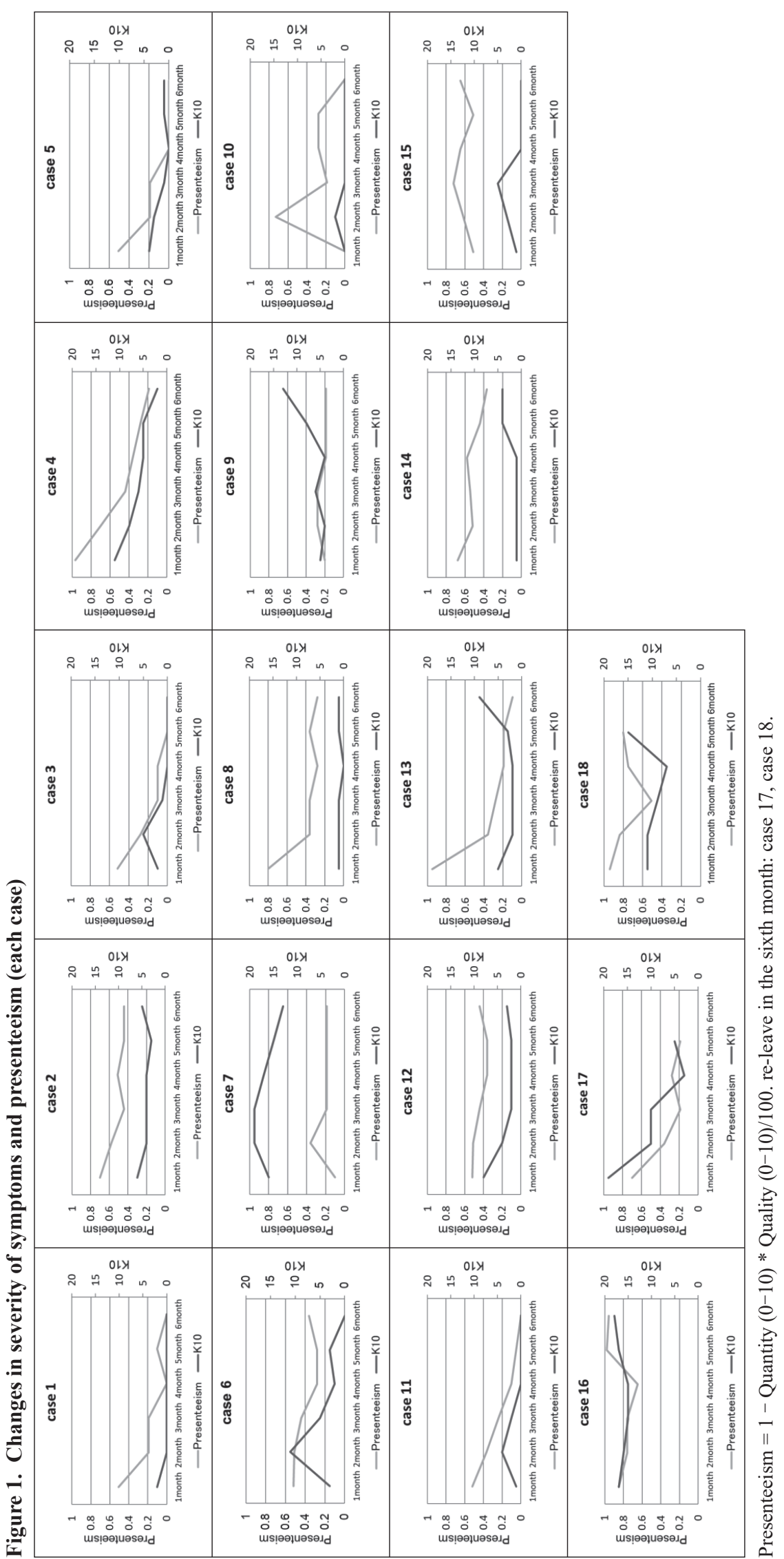


cutoff, presenteeism at the sixth month was 0 in 5 employees (cases 1, 3, 5, 10, and 11), and greater than 0 in 6 employees (cases 2, 8, 12, 13, 14, and 15). Among the five employees in which $\mathrm{K} 10$ exceeded the cutoff value at least once during the six months (cases 4, 6, 7, 9, and 16), not a single case of presenteeism resulted in 0 (Figure 1).

\section{Presence of the OP's written opinion}

The OP did not issue an opinion recommending work restriction at the sixth month after RTW for four employees (cases 1, 3, 5, and 10) (Table 6). The value of presenteeism at the sixth month was 0 for all four of those employees. The work restriction was not lifted in case $11(\mathrm{~K} 10=0$, presenteeism $=0$ at the sixth month).

\section{Discussion}

\section{Monthly changes in psychological distress}

The results revealed that the average psychological distress (K10) was below the cutoff value of 10 [17]. Although there was an overall decreasing trend, there was no difference between the first month and each other month, probably because psychological distress improved throughout all the months and did not dif-

Table 5. Changes in presenteeism after returning from sick leave due to mental illness

\begin{tabular}{lllllll}
\hline & & Coefficient & Standard Error & $95 \%$ Confidence Interval & $P$ \\
\hline month(continuous) & & -0.06 & 0.01 & -0.07 & -0.04 & 0.000 \\
month(categorical) & 1 & $\begin{array}{l}\text { reference } \\
\end{array}$ & & & & \\
& 2 & -0.10 & 0.05 & -0.19 & 0.00 & 0.051 \\
& 3 & -0.19 & 0.05 & -0.29 & -0.09 & 0.000 \\
& 4 & -0.26 & 0.05 & -0.36 & -0.16 & 0.000 \\
& 5 & -0.26 & 0.05 & -0.36 & -0.17 & 0.000 \\
& 6 & -0.29 & 0.05 & -0.39 & -0.20 & 0.000 \\
\hline
\end{tabular}

Table 6. Status of work restrictions after RTW

\begin{tabular}{lllllll}
\hline & at 1 month & at 2 month & at 3 month & at 4 month & ata 5 month & at 6 month \\
\hline case 1 & yes & yes & yes & no & no & no \\
case 2 & yes & yes & yes & yes & yes & yes \\
case 3 & yes & yes & yes & yes & yes & no \\
case 4 & yes & yes & yes & yes & yes & yes \\
case 5 & yes & yes & no & no & no & no \\
case 6 & yes & yes & yes & yes & yes & yes \\
case 7 & yes & yes & yes & yes & yes & yes \\
case 8 & yes & yes & yes & yes & yes & yes \\
case 9 & yes & yes & yes & yes & yes & yes \\
case 10 & yes & yes & yes & yes & no & no \\
case 11 & yes & yes & missing & yes & missing & yes \\
case 12 & yes & yes & yes & yes & yes & yes \\
case 13 & yes & yes & missing & yes & yes & yes \\
case 14 & yes & yes & missing & yes & yes & yes \\
case 15 & yes & missing & yes & yes & yes & yes \\
case 16 & yes & missing & yes & yes & yes & yes \\
case 17 & yes & yes & yes & yes & yes & sick leave \\
case 18 & yes & yes & yes & yes & yes & sick leave \\
\hline
\end{tabular}

RTW: return to work 
fer enough to be significant. However, changes in the $\mathrm{K} 10$ values varied between cases. Regarding the transition of symptoms of depression, Greco et al reported that there is a steep improvement in the first month of treatment, which becomes gradual thereafter [19], but our study was based on data from the start of RTW and not from the start of treatment. Of the 16 employees who did not take additional leave of absence, 11 of those who did not exceed the cutoff value at any time in the six months were continuously mentally stable after RTW. Of the five who exceeded the cutoff at least once, however, two (cases 7 and 16) were consistently above the cutoff during the six months. There were also employees in whom the K10 increased later in the observation period (including case 18, who took a leave of absence again); these employees appeared to require attention.

\section{Monthly changes in presenteeism}

Overall, our results indicated that presenteeism was high in the first month after RTW, with a tendency to decrease after the second month. Individually, of the 11 employees in which the K10 did not exceed the cutoff value, presenteeism was 0 in 5 employees (cases $1,3,5,10$, and 11) at the sixth month, reflecting an overall decreasing pattern. The six employees (cases $2,8,12,13,14$, and 15) in which presenteeism at the sixth month was greater than 0 exhibited a pattern of stopping the decline midway and plateauing, even if the value of presenteeism decreased.

Conversely, in the five employees in which the K10 exceeded the cutoff value at least once during the six months (cases 4, 6, 7, 9, and 16), a decreasing pattern (case 4) and a plateau pattern (cases 6, 7, and 9), and a case in which presenteeism remained high for the study period (case 16) were found. Although no previous study has examined the transition of presenteeism after RTW, some reports have indicated that presenteeism persists even when symptoms improve [10-12]. In the present study, although not all employees with stable psychological distress showed a delay in the improvement of presenteeism, two patterns of change of presenteeism were observed: one in which presenteeism gradually declined and one in which it stopped declining and plateaued.

Cohen et al reported that remission in depressed pa- tients, as defined by a reduction in symptom severity, did not denote normal functioning [20]. Hirschfeld et al reported that not all treatments are equally effective in relieving the impaired social functioning associated with depressive disorders and that efficacy in relieving the core symptoms of depression does not necessarily guarantee efficacy in relieving impaired social functioning [21].

Toyoshima et al reported that complaints of cognitive functions (CCFs), defined as subjective cognitive dysfunction, significantly mediate the associations among insomnia, state of anxiety, and presenteeism, and pointed out that it may be useful to assess the mediating roles of CCFs to address the presenteeism associated with insomnia and state of anxiety [22]. Toyoshima et al also reported that CCFs mediate the relationship between depressive symptoms and presenteeism in the following manner: "depressive symptoms $\rightarrow$ CCFs $\rightarrow$ presenteeism” [23].

Cases in which presenteeism stopped improving while K10 improved were considered to have social dysfunction or cognitive dysfunction separate from the severity of the symptoms. The relationship among psychiatric symptoms, social dysfunction, cognitive dysfunction, and presenteeism requires further study.

\section{Relationship between the OP's opinions and presen-} teeism

The OP's opinions were based on the interview with workers who had returned to work. During the interview, the worker answered the K10 and presenteeism questionnaires, therefore the OP's opinions were considered to reflect the results of those questionnaires. The work restriction was lifted when the value of the K10 improved and presenteeism was zero, according to the OP's opinion, at the sixth month after RTW, except for case 11 . The concern of the OP regarding case 11 was unclear.

OPs' opinions submitted to the workplace are based on a variety of considerations, and opinions are not meant to be an objective assessment of presenteeism. After RTW, the OPs often take steps to remove restrictions in stages, based not only on psychiatric symptoms, but also on whether the employee is readjusting to work. Namba reported the importance of adjusting the workload in stages after RTW [24]. This gradual 
adjustment of the workload is considered to be consequently associated with not only psychiatric symptoms but also the existence of presenteeism as a state of decreased productivity due to poor physical or health condition.

Mori mentioned the need to consider how presenteeism can be dealt with on an individual basis, as there are different backgrounds and causes for presenteeism for each person [25]. It is necessary to examine how OPs can help improve presenteeism in the future, and one way to confirm this is to check the OP's opinion.

\section{Limitations}

An important limitation of the current study is the relatively small number of cases, and the fact that this study was conducted in a single company by a single OP. The respondents to the survey were all workers who underwent monthly interviews with an OP for six months after RTW. We used a study period of six months following Norder's recommendation [15], but a six-month study period is relatively short; Endo et al recommended that relapse prevention consultations should be taken for up to five years [26]. Without intervention in the long term, presenteeism may continue, potentially resulting in the need for more sick leave.

This study collected data only in the context of monthly OP interviews, which may have had a significant impact on the results of psychological distress and presenteeism. It did not check whether an RTW support system such as "Rework" was used before RTW [27], or whether the workplace after RTW was the same or different compared with before the sick leave. The extent to which these factors may have affected the results remains unclear.

It was also impossible to obtain complete monthly interview data for all the subjects. Because the data set would have been smaller if only subjects with complete data were included ( $\mathrm{n}=11$ ), we adopted missing imputation and used the last observation carried forward to present the statistics. Checking the mean assignment method for both $\mathrm{K} 10$ and presenteeism revealed that they had no effect on the results. Aggregate values using this method are presented because they do not affect the overall average.

Despite these limitations, the study has certain strengths. The survey checked psychological distress and presenteeism in monthly interviews, and the recall bias was low. Because the diagnosis was confirmed by a medical certificate provided by a psychiatrist, the diagnosis was likely to be relatively accurate. No previous study has confirmed the relationship between presenteeism and psychological distress every month for six months; thus the findings of the present study extend previous research in a meaningful way. In future, it will be important to develop better methods to support OP's interviews with workers with mental illness after RTW to improve presenteeism gradually without exacerbating symptoms.

\section{Conclusion}

Sixteen workers with mental illness were examined for changes in psychological distress and presenteeism six months after RTW. Two employees took sick leave again in the sixth month. Eleven of the 16 employees were consistently below the K10 cutoff point for six months, and 5 had 0 presenteeism in the sixth month; 6 employees, however, exhibited improvement in presenteeism that stopped midway through the study. In the five employees in which the K10 exceeded the cutoff value at least once during six months, there was not a single month in which presenteeism was zero. Cases in which presenteeism stopped improving while K10 improved were considered to have social dysfunction separate from the severity of the symptoms. The OP's opinions regarding work restriction were found to reflect not only psychological distress but also the results of presenteeism. In future, it will be necessary to further investigate the trends of presenteeism after RTW to develop approaches for gradually reducing presenteeism.

\section{Acknowledgments}

The authors would like to express their sincere thanks to all the participants in this study.

\section{Conflict of Interest}

The authors declare no conflicts of interest associated with this manuscript. 


\section{References}

1. Goetzel RZ, Long SR, Ozminkowski RJ, Hawkins K, Wang S \& Lynch W (2004): Health, absence, disability, and presenteeism cost estimates of certain physical and mental health conditions affecting U.S. employers. J Occup Environ Med 46(4): 398-412

2. Loeppke R, Taitel M, Haufle V, Parry T, Kessler RC \& Jinnett K (2009): Health and productivity as a business strategy: a multiemployer study. J Occup Environ Med 51(4): 411-428

3. Wada K, Arakida M, Watanabe R, Negishi M, Sato J \& Tsutsumi A (2013): The economic impact of loss of performance due to absenteeism and presenteeism caused by depressive symptoms and comorbid health conditions among Japanese workers. Ind Health 51(5): 482-489

4. Nagata T, Mori K, Ohtani M et al (2018): Total HealthRelated Costs Due to Absenteeism, Presenteeism, and Medical and Pharmaceutical Expenses in Japanese Employers. J Occup Environ Med 60(5): e273-e280

5. Ministry of Health Labor and Welfare (2013): Guidelines of support of RTW of workers absent with mental health problem. https://www.mhlw.go.jp/content/ 000561013.pdf (accessed May 27, 2021) (in Japanese)

6. Hori H, Katsuki A, Atake K, Yoshimura R, Nakamura J \& Baune BT (2019): Risk factors for further sick leave among Japanese workers returning to work after an episode of major depressive disorder: a prospective follow-up study over 1 year. BMJ Open 9(9): e029705

7. Noben CY, Evers SM, Nijhuis FJ \& de Rijk AE (2014): Quality appraisal of generic self-reported instruments measuring health-related productivity changes: a systematic review. BMC Public Health 14: 115

8. Ospina MB, Dennett L, Waye A, Jacobs P \& Thompson AH (2015): A systematic review of measurement properties of instruments assessing presenteeism. Am J Manag Care 21(2): e171-e185

9. Johns G (2010): Presenteeism in the workplace: a review and research agenda. J Organ Behav 31(4): 519542

10. Zhang W, Bansback N \& Anis AH (2011): Measuring and valuing productivity loss due to poor health: a critical review. Soc Sci Med 72(2): 185-192

11. Fujino Y, Uehara M, Izumi H et al (2015): Development and validity of a work functioning impairment scale based on the Rasch model among Japanese workers. J Occup Health 57(6): 521-531

12. Adler DA, McLaughlin TJ, Rogers WH, Chang H, Lapitsky L \& Lerner D (2006): Job performance deficits due to depression. Am J Psychiatry 163(9): 15691576

13. Curkendall S, Ruiz KM, Joish V \& Mark TL(2010): Productivity losses among treated depressed patients relative to healthy controls. J Occup Environ Med 52(2): 125-130

14. Gilmour H \& Patten SB (2007): Depression and work impairment. Health Rep 18(1): 9-22

15. Norder G, Hoedeman R, de Bruin J, van Rhenen W \& Roelen CA (2015): Time to recurrence of mental health-related absence from work. Occup Med (Lond) 65(7): 574-577

16. Kessler RC, Andrews G, Colpe LJ et al (2002): Short screening scales to monitor population prevalences and trends in non-specific psychological distress. Psychol Med 32(6): 959-976

17. Sakurai K, Nishi A, Kondo K, Yanagida K \& Kawakami N (2011): Screening performance of K6/K10 and other screening instruments for mood and anxiety disorders in Japan. Psychiatry Clin Neurosci 65(5): 434441

18. Brouwer WB, Koopmanschap MA \& Rutten FF (1999): Productivity losses without absence: measurement validation and empirical evidence. Health Policy 48(1): $13-27$

19. Greco T, Eckert G \& Kroenke K (2004): The outcome of physical symptoms with treatment of depression. J Gen Intern Med 19(8): 813-818

20. Cohen RM, Greenberg JM \& IsHak WW (2013): Incorporating multidimensional patient-reported outcomes of symptom severity, functioning, and quality of life in the Individual Burden of Illness Index for Depression to measure treatment impact and recovery in MDD. JAMA Psychiatry 70(3): 343-350

21. Hirschfeld RM, Montgomery SA, Keller MB et al (2000): Social functioning in depression: a review. J Clin Psychiatry 61(4): 268-275

22. Toyoshima K, Inoue T, Shimura A et al (2021): Mediating roles of cognitive complaints on relationships between insomnia, state anxiety, and presenteeism in Japanese adult workers. Int J Environ Res Public Health 18(9): 4516 
23. Toyoshima K, Inoue T, Shimura A et al (2020): Associations between the depressive symptoms, subjective cognitive function, and presenteeism of Japanese adult workers: a cross-sectional survey study. Biopsychosoc Med 14: 10

24. Namba K (2012): A Return-to-work program with a relapse-free job retention rate of $91.6 \%$ for workers with mental illness. Sangyo Eiseigaku Zasshi 54(6): $276-285$

25. Mori K(2019): Chapter 5 Supportive and Promotive Occupational Health Programs. In: How to Promote Health Management for Better Results. Rodochousakai, To- kyo pp 145-173 (in Japanese)

26. Endo M, Haruyama Y, Muto T, Yuhara M, Asada K \& Kato R (2013): Recurrence of sickness absence due to depression after returning to work at a Japanese IT company. Ind Health 51(2): 165-171

27. Japanese Association of Rework for Depression: Depression Rework Research Association Manual of Rework for Therapists. http:/www.utsu-rework.org/info/ cstaff/manual.pdf (accessed May 27, 2021) (in Japanese)

J UOEH $43(4): 385-395(2021)$ 